\title{
Upshaw-Schulman Syndrome
}

\author{
Wg Cdr BM John*, Gp Capt D Singh+, Gp Capt B Ravichander (Retd), Wg Cdr R Madan**, \\ Air Vice Mshl (Retd) TS Raghu Raman, AvSM $^{++}$
}

MJAFI 2010; 66 : 188-189

Key Words : Congenital thrombotic thrombocytopenic purpura; von Willebrand factor cleaving protease

\section{Introduction}

$\mathrm{T}_{\mathrm{c}}^{\mathrm{h}}$ hrombotic thrombocytopenic purpura (TTP) is characterized by microangiopathic haemolytic anaemia and thrombocytopenia, often accompanied by fever, renal failure and neurological deficits. The pathophysiology of TTP has been mysterious until recently. Unusually large von Willebrand factor (ULVWF) multimers are now being incriminated in causation of TTP by promoting uncontrolled platelet agglutination, thrombosis, and ischemia. Congenital TTP is associated with a deficiency in a plasma metalloprotease that cleaves a specific peptide bond in the von Willebrand factor (VWF) subunit, thereby decreasing the size of VWF multimers, while acquired TTP seen more often in adolescents and adults is associated with auto-antibodies neutralizing the metalloprotease [1-2]. Here, we report a child with congenital TTP or Upshaw-Schulman syndrome.

\section{Case Report}

An eight-year-old boy born of a third degree consanguineous marriage presented to us with petechiae, pallor and jaundice. His medical documents revealed that he had first manifested with petechiae and pallor at five years of age and investigations showed thrombocytopenia with schistocytic anaemia. The other investigations done were renal function tests which were normal and Coomb's test which was negative. He responded to a single whole blood transfusion and was discharged without a definite diagnosis. Thereafter, he had three episodes of petechiae associated with thrombocytopenia without anaemia between the age of six to eight years during which a diagnosis of ITP and subsequently of chronic ITP was made and child received steroids. Bone marrow evaluation done at that point was reported as normal. On presentation to us, he had normal vital parameters, had no positive systemic findings and the investigations revealed haemoglobin $(\mathrm{Hb}) 6 \mathrm{~g} / \mathrm{dl}$, total lecuocyte count (TLC) 15700/cumm (P 69\%), platelet count $15000 /$ cumm, corrected reticulocyte count $-15 \%$ and peripheral blood smear (PBS) suggestive of microangiopathic haemolytic anaemia with schistocytes and thrombocytopenia. Urine routine and microscopic examination were normal. Liver function test (LFT) revealed unconjugated bilirubinemia (bilirubin $3.8 \mathrm{mg} / \mathrm{dl}$, unconjugated $3.2 \mathrm{mg} / \mathrm{dl}$ ) with normal protein and enzyme levels. Prothrombin time $13 \mathrm{sec}$ (corrected $12 \mathrm{sec})$ and activated partial thromboplastin time $34 \mathrm{sec}$ (corrected $32 \mathrm{sec}$ ) were normal. The blood urea nitrogen / creatinine and serum electrolytes were normal. The lactate dehydrogenase (LDH) level was elevated to 1014 U/L. Serum haptoglobin was low at $5 \mathrm{mg} / \mathrm{dl}$ (normal range $30-200 \mathrm{mg} / \mathrm{dl}$ ). The tests for human immunodeficiency virus (HIV), glucose 6 phosphodehydrogenase (G6PD) screen, indirect and direct Coomb's test, antinuclear antibody and anticardiolipin antibody were negative. C3 levels of $80 \mathrm{mg} / \mathrm{dl}$ and serum ceruloplasmin $36 \mathrm{mg} / \mathrm{dl}$ were normal. The neonatal period had been uneventful. The parental and sibling screen with hemogram and peripheral blood smear were normal. Based on the overall history and clinical profile of this schistocytic anaemia with thrombocytopenia in a relatively well child, a diagnosis of congenital TTP was considered and child given transfusion with packed red blood cells (RBC) and fresh frozen plasma (FFP). His thrombocytopenia corrected with this management (platelet count-1.6 lacs/cumm). On follow up at four weeks, a sample sent for von Willebrand factor multimer analysis by SDS-agarose gel electrophoresis showed unusually large VWF multimers. He received another FFP after two-months when there was an evidence of thrombocytopenia (platelet count increased from 60000/cumm to 1.9 lacs/cumm after FFP), and discharged with a plan for periodic FFP transfusions, pending the availability of ADAMTS 13 assay in our country.

\section{Discussion}

The classical description of TTP consists of the pentad of microangiopathic haemolytic anaemia (MAHA),

${ }^{*}$ Reader (Dept of Paediatrics), AFMC, Pune-40. ${ }^{+}$Senior Advisor (Paediatrics \& Neonatology), Command Hospital (SC), Pune-40. ${ }^{*}$ Professor \& HOD (Paediatrics), MVJ Medical College, Bangalore. ${ }^{* *}$ Classified Specialist (Pathology), Army Hospital (R\&R), New Delhi. ${ }^{++}$Medical Superintendent, Kasturba Hospital, Manipal-576104, Karnataka.

Received : 25.03.08; Accepted : 22.02.10_E-mail : drbmj1972@yahoo.com 
thrombocytopenia, fever, renal and neurological dysfunction. However, this pentad is present in only $40 \%$ of cases. Some workers have proposed diagnosis based on the presence of at least three of the following: thrombocytopenia, haemolytic anaemia, elevated serum levels of lactate dehydrogenase and neurological symptoms $[1,3]$. TTP should be considered in any condition with MAHA and thrombocytopenia with or without renal or neurological dysfunction, without any other obvious cause [4-5].

Patients with congenital TTP are deficient in a plasma metalloprotease (ADAMTS 13) which belongs to the ADAMTS (an acronym for a disintegrin and metalloprotease with thrombospondin-1-like domains) family of metalloproteases that cleaves a specific peptide bond in the VWF subunit, thereby decreasing the size of VWF multimers. The deficiency of ADAMTS 13 results in unusually large VWF multimers (ULVWF) causing uncontrolled platelet aggregation and microvascular thrombosis [6-7]. TTP may be favoured by conditions that combine increased VWF secretion and decreased ADAMTS 13 activity. Such a "two-hit" model could explain the substantial variation in the age at which patients with inherited TTP develop symptoms. Thrombotic microangiopathy may supervene after a triggering event injures or activates microvascular endothelial cells and causes the secretion of ULVWF against a background of ADAMTS 13 deficiency [6].

In hereditary TTP, first manifestations occur before the age of ten years in two thirds of cases. The subsequent course is characterized by recurrent haemolytic and thrombocytopenic crises, with intervals between relapses from every 3 to 4 weeks in two thirds of cases to several months or years in one third of cases. TTP crises are associated with cerebral vascular accidents in at least $30 \%$ of patients. Renal involvement includes frequent acute renal failure due to hemoglobinuria and/or thrombotic microangiopathy during haemolytic crisis and progressive renal deterioration in approximately $50 \%$ of cases. Some mild forms present with only fluctuating thrombocytopenia and may be misdiagnosed as ITP like in our case [3]. The other causes of schistocytic anaemia with thrombocytopenia include haemolytic uremic syndrome, malignancy, drugs, giant vascular malformations, preeclampsia/ eclampsia and immunologic vasculitidis [1-3].

Genetic analyses of patients with familial TTP have linked the disease to chromosome $9 \mathrm{q} 34$ and an increased incidence is seen in people with HLA-B40 group antigens [8]. The diagnosis which is based on assay of the ADAMTS 13 protease (levels $<5-10 \%$ considered suggestive of deficiency) is complicated and is unavailable in our country. The plasma concentration of ADAMTS 13 which is mostly synthesized in the liver is estimated to be $1 \mu \mathrm{g} / \mathrm{ml}$. Its half-life in the circulation is approximately 2 to 3 days and this stability allows patients with congenital deficiency to be treated with plasma infusions every 2 to 3 weeks to prevent the recurrence of thrombotic microangiopathy [6]. FFP infusions are thought to be best used preventively, given that rescue infusions may not prevent central nervous system and renal involvement. It is hoped that plasma or recombinant purified ADAMTS 13 will be available in the future [3].

Our case, which was initially diagnosed as ITP, came to light with his presentation with MAHA and thrombocytopenia. In the absence of availability of ADAMTS 13 protease estimation in our country, the clinical response to FFP and presence of ULVWF on VWF multimer analysis were taken to be diagnostic of congenital TTP. This unusual case of congenital TTP with recurrent schistocytic haemolytic anaemia and thrombocytopenia without renal, neurological or any other systemic involvement has been brought out to emphasize the expanding phenotype of hereditary TTP. With the understanding of the role of ADAMTS 13 protease, the disease is being diagnosed more often. Paediatricians should be aware of this condition, given that effective treatment is available.

\section{Conflicts of Interest}

None identified

\section{References}

1. Lammle B, Kremer HJA, Alberio LJ. Thrombotic thrombocytopenic purpura. Thromb Haemost 2005; 3: 166375.

2. George JN. Congenital Thrombotic Thrombocytopenic Purpura: Lessons for recognition and management of rare syndromes. Paediatr Blood Cancer 2008;50:947-8.

3. Loirat C, Veyradier A, Girma JP, Ribba AS, Meyer D. Thrombotic thrombocytopenic purpura associated with von Willebrand factor-cleaving protease (ADAMTS13) deficiency in children. Semin Thromb Hemost 2006; 32: 90-7.

4. Veyradier A, Meyer D. Thrombotic thrombocytopenic purpura and its diagnosis. J Thromb Haemost 2005; 3:2420-7.

5. George JN. Thrombotic thrombocytopenic purpura. N Engl J Med 2006; 354:1927-35.

6. George J N, Sadler J E, Lämmle B. Platelets: Thrombotic Thrombocytopenic Purpura. Hematology Am Soc Hematol Educ Program 2002; 1: 315-34.

7. Jubinsky P T, Moraille R, Tsai Han-Mou. Thrombotic Thrombocytopenic Purpura in a Newborn. J Perinatol 2003 ; 23: 85-7.

8. Rodrigues RG. Two generations with familial thrombotic thrombocytopenic purpura. Int J Clin Pract 2006; 60: 95-8. 